\title{
Endocrine-related adverse events in a large series of cancer patients treated with anti-PD1 therapy
}

\author{
Rossella Rubino ${ }^{1}$ - Andrea Marini ${ }^{2}$ - Giandomenico Roviello ${ }^{2}$ - Elena Margherita Presotto ${ }^{1}$ Isacco Desideri ${ }^{3}$.

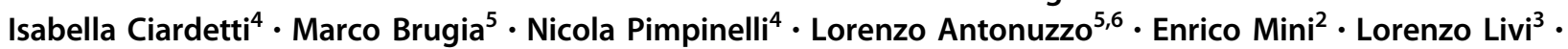

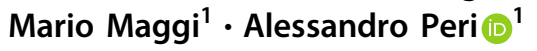

Received: 7 January 2021 / Accepted: 3 May 2021 / Published online: 25 May 2021

(c) The Author(s) 2021

\begin{abstract}
Purpose Immune checkpoint inhibitors have opened a new scenario in the treatment of cancer. These agents can elicit adverse events, which may affect different systems and organs, including the endocrine system. The aims of this study were to evaluate the impact of the anti-PD-1 molecules nivolumab and pembrolizumab on endocrine toxicity and on patient outcome.

Methods A retrospective and multicentre study was designed, which involved a total of 251 patients affected by different tumors (mostly non-small cell lung cancer, $68.92 \%$ and melanoma, 24.30\%) and treated with the PD-1 inhibitors nivolumab $(61.35 \%)$ or pembrolizumab (38.65\%) for up to 60 months. Clinical and biochemical data were recorded until July 31, 2020. Results Endocrine toxicity occurred in 70 out of 251 patients $(27.89 \%)$. It was mostly related to thyroid dysfunction and in $75 \%$ of cases occurred within 6 months from the beginning of therapy. A previous endocrine morbidity and female gender were predictors of endocrine toxicity. There was no association between endocrine dysfunction and patient outcome. However, when all toxicities (i.e., endocrine and non endocrine) were considered, a significant association with progressionfree survival and overall survival was found.

Conclusions Thyroid alterations are frequently observed in cancer patients treated with anti PD-1 drugs, particularly in women and in the presence of a previous endocrinopathy. We suggest that regular thyroid assessment should be performed in these patients, especially in the first months of therapy. Finally, the onset of side effects, related to anti PD-1 agents, appears to be associated with a better outcome.
\end{abstract}

Keywords Endocrine toxicity $\cdot$ Cancer $\cdot$ Immunotherapy $\cdot$ Anti PD-1

Alessandro Peri

alessandro.peri@unifi.it

1 Endocrine Unit, Department of Experimental and Clinical Biomedical Sciences "Mario Serio", University of Florence, AOU Careggi, Florence, Italy

2 Unit of Translational Oncology, AOU Careggi, Department of Health Sciences, Section of Clinical Pharmacology and Oncology, University of Florence, AOU Careggi, Florence, Italy

3 Radiation Oncology Unit, Oncology Department, AOU Careggi, Department of Experimental Clinical and Biomedical Sciences "Mario Serio", University of Florence, Florence, Italy

4 Melanoma \& Skin Cancer Unit, Tuscany Central District; Department of Health Sciences, Dermatology Unit, University of Florence, Florence, Italy

5 Medical Oncology Unit, AOU Careggi, Florence, Italy

6 Department of Experimental and Clinical Medicine, University of Florence, Florence, Italy

\section{Introduction}

Immune checkpoint inhibitors (ICPIs) opened a new era in the treatment of advanced cancer. ICPIs are monoclonal antibodies that inhibit negative regulatory pathways of the immune response, ultimately stimulating $\mathrm{T}$ cells to attack cancer cells. The $\mathrm{T}$ lymphocytes-associated antigen 4 (CTLA-4), the programmed cell death protein-1 (PD-1) and its ligands (PD-L1/PD-L2) are the targets that have been utilized in recent years, in order to design new pharmacological strategies [1]. Historically, the CTLA-4 inhibitor ipilimumab was the first ICPI approved in the U.S. for the treatment of advanced-stage melanoma. PD-1 inhibitors (e.g., nivolumab, pembrolizumab) and PD-L1 inhibitors (e.g., atezolizumab, avelumab, durvalumab) followed and the spectrum of indications has widened to different tumors, including, for instance, non-small cell lung cancer (NSCLC), 
Table 1 Demographic characteristics of the patients

\begin{tabular}{llll}
\hline & Males & Females & Total \\
\hline Number & 155 & 96 & 251 \\
Age (years) & $\begin{array}{l}71.63 \pm 1.57 \\
(\text { range } 46-93)\end{array}$ & $\begin{array}{l}68.93 \pm 2.15 \\
(\text { range } 44-95)\end{array}$ & $\begin{array}{l}70.60 \pm 1.28 \\
\text { (range 44-95) }\end{array}$ \\
Tumor & & & \\
NSCLC & $109(70.32 \%)$ & $64(66.66 \%)$ & $173(68.92 \%)$ \\
Melanoma & $38(24.52 \%)$ & $23(23.96 \%)$ & $61(24.30 \%)$ \\
Kidney & $7(4.52 \%)$ & $3(3.12 \%)$ & $10(3.98 \%)$ \\
Breast & 0 & $4(4.16 \%)$ & $4(1.59 \%)$ \\
Head-Neck & $1(0.64 \%)$ & $1(1.04 \%)$ & $2(0.80 \%)$ \\
Bladder & 0 & $1(1.04 \%)$ & $1(0.40 \%)$ \\
ICPI & $94(60.64 \%)$ & $60(62.50 \%)$ & $154(61.35 \%)$ \\
Nivolumab & $61(39.35 \%)$ & $36(37.50 \%)$ & $97(38.65 \%)$ \\
Pembrolizumab & & &
\end{tabular}

renal cell carcinoma, Hodgkin lymphoma, head and neck squamous cell cancer, hepatocellular carcinoma [2-9].

Because of their mechanism of action, ICPIs can be associated with immune-related adverse events (irAEs). The most commonly reported irAEs involve the respiratory and gastrointestinal apparatus, the skin, the endocrine glands (e.g., pituitary, thyroid, adrenal, endocrine pancreas). Hypophysitis can occur during immunotherapy and is more commonly observed in patients treated with ipilimumab $[10,11]$. Thyropathies are more frequently associated with anti-PD-1 treatment or with a combination of anti-PD-1 and anti-CTLA4 [10, 11]. Insulin-deficient diabetes mellitus and primary adrenal insufficiency are rarely reported $[10,11]$. With regard to thyroid dysfunction, which is overall the most frequent endocrine irAE, both hypothyroidism or thyrotoxicosis may occur. The latter can be transient and can be followed by subsequent hypothyroidism [12,13].

The aim of this retrospective, multicentre study was to analyze: (i) the occurrence and the course of endocrine irAEs in a series of 251 patients with different cancers treated with the PD-1 inhibitors nivolumab or pembrolizumab, and (ii) the relationship between the onset of irAEs and outcome.

\section{Patients and methods}

\section{Patients}

Two hundred and fifty-one patients consecutively referred to reference Centres in the Florence area (e.g., Radiation Oncology Unit, Medical Oncology Unit and Unit of Translational Oncology at the AOU Careggi; Melanoma \& Skin Cancer Unit, Tuscany Central District, IOT Hospital) and treated with nivolumab or pembrolizumab for advanced cancer disease were enrolled in this retrospective study.
Consent for the data collection was collected from each patient. The study was approved by the Careggi Hospital Ethics Committee. Clinical data from October 1, 2017 to July 31,2020 were recorded for patients with a follow-up of at least 2 months from the initiation of immunotherapy. The followup ranged from 2 to 60 months. Nivolumab was administered i.v. (usually $3 \mathrm{mg} / \mathrm{kg}$ ) every 2 weeks, pembrolizumab was administered i.v. (usually $2 \mathrm{mg} / \mathrm{kg}$ ) every 3 weeks. Table 1 shows the demographic characteristics of the patients. Biochemical data, including thyroid function, cortisol, glycemia, serum electrolytes at baseline and during ICPIs therapy were collected from clinical charts. All the hormone and biochemistry measurements were performed at the central lab of the Careggi Hospital. Normal plasma levels were within $0.36-3.74 \mathrm{mU} / \mathrm{L}$ for TSH, $9.8-18.8 \mathrm{pmol} / \mathrm{L}$ for fT4, $3.3-6.1 \mathrm{pmol} / \mathrm{L}$ for $\mathrm{fT} 3,138-635 \mathrm{nmol} / \mathrm{L}$ for cortisol, $65-110 \mathrm{mg} / \mathrm{dL}$ for glycemia. Blood samples had been drawn after an overnight fast before every treatment with anti-PD-1. All the blood parameters were measured according to a routinary screening of possible complications of ICPIs treatment. In patients who were receiving corticosteroids at some point during follow up (about $15-20 \%$, yet only $3-4 \%$ of cases at high doses for the treatment of severe toxicities), blood serum cortisol was assessed once treatment had been discontinued.

\section{Statistical analysis}

Continuous variables with a normal distribution and categorical variables were expressed as mean $\pm 95 \%$ confidence interval. Differences between groups in continuous and categorical variables were assessed by Student $t$ and chisquared test, respectively. The simultaneous analysis of all possible predictors of endocrine toxicity was performed by binary logistic regression. The analysis aimed to assess the possible association between five putative predictors and the occurrence of endocrine alterations, with "no endocrine toxicity" or "endocrine toxicity" being the dependent variable.

The trend over time of thyroid toxicity was evaluated by a survival analysis using the log-rank test to evaluate the difference in events among the months of observation.

The correlation between the onset of toxicities and outcome was analyzed using chi-squared test. Progression free survival (PFS) and overall survival (OS) were represented using Kaplan-Meier analysis and compared using log-rank test.

\section{Results}

\section{Endocrine Toxicity}

Endocrine irAEs were registered in 70 out of 251 patients $(27.89 \%)$. Of these 70 patients, 36 were males $(51.43 \%)$ and $34(48.57 \%)$ were females. The follow up ranged from 
Table 2 Patients on follow-up at different times

\begin{tabular}{ll}
\hline Months & N. of patients \\
\hline 1 & 251 \\
2 & 251 \\
3 & 246 \\
4 & 237 \\
5 & 226 \\
6 & 215 \\
12 & 182 \\
24 & 99 \\
36 & 48 \\
48 & 18 \\
60 & 5 \\
\hline
\end{tabular}

2 months to 60 months (median $18.48 \pm 1.7,95 \% \mathrm{CI}$ ) (Table 2). With regard to the type of tumor in patients with endocrine toxicity, 53 (75.71\%) patients had NSCLC, 13 (18.57\%) melanoma, 2 (2.85\%) renal cancer, 1 (1.43\%) breast cancer and 1 (1.43\%) bladder cancer. Forty-two $(60 \%)$ of these patients were treated with nivolumab and 28 (40\%) with pembrolizumab. In the large majority of cases, endocrine toxicity was related to a thyropathy $(n=66$, 94.28\%). It is known that also tyrosine kinase inhibitors, which may precede immunotherapy, may be associated with thyroid dysfunction or may affect the onset of ICPI related toxicity $[14,15]$. However, in our series, only 6 patients had been previously treated with tyrosine. Kinase inhibitors and thyroid alterations had not been reported. Besides thyroid dysfunction, in two patients $(2.86 \%)$ the onset of type 1 diabetes mellitus was reported and insulin treatment was started. In two other cases $(2.86 \%)$ secondary hypocortisolism or hypothyroidism were observed.

The analysis of the presence of possible risk factors for the development of endocrine irAEs showed that a preexisting endocrinopathy was a strong predictor (Table 3). A gender difference was also observed, namely women had a higher risk to develop an endocrinopathy during treatment with nivolumab or pembrolizumab than men. Conversely, the type of cancer, the type of anti PD-1 and age were not predictors of endocrine toxicity in our series of patients. Moreover, a pre-existing endocrinopathy was confirmed as a significant predictive factor even when analyzed separately in males and females (not shown).

The above-mentioned predictors were further analyzed simultaneously in a unique statistical model using the binary logistic regression in order to assess a possible interaction between each predictor. The results showed that a pre-existing endocrinopathy remained a significant predictor, with a $53.9 \%$ probability of occurrence of endocrine toxicity during immunotherapy (Table 4).
In 33 patients $(47.14 \%)$ endocrine toxicity was present within 2 months from the initiation of immunotherapy and in $53(75.71 \%$ ) within the first 6 months (Fig. 1). No difference in the time of appearance of toxicity was observed between patients with or without a pre-existing endocrinopathy $(15.14 \pm 5.92$ vs $16.81 \pm 4.55$ weeks, respectively, $p>0.05)$.

\section{Thyropathy}

Among the 66 patients who presented a thyropathy, 38 $(73.68 \%)$ had no previous history of endocrinopathies, whereas $28(26.32 \%)$ had already a previous endocrine disease (i.e., a prexisting thyroid alteration or diabetes mellitus). The most frequent thyroid alteration was hypothyroidism $(n=34,51.52 \%)$, followed by thyrotoxicosis $(n=17,25.76 \%)$ and transient thyrotoxicosis followed by hypothyroidism $(n=15,22.72 \%)$. In more than half of patients $(n=36,54.5 \%)$ thyroid alteration was mild and did not require specific pharmacological intervention, whereas in 30 cases (45.5\%) thyroid specific treatment was started or dose adjustment was needed.

\section{irAEs and outcome}

In order to assess whether endocrine toxicity was associated with a different outcome in our series of cancer patients treated with nivolumab or pembrolizumab, Kaplan-Meier estimates were analyzed in a homogeneous subgroup of patients with NSCLC $(n=92)$, who had started immunotherapy by December 31, 2018 and had received at least four treatment cycles. Neither PFS, nor OS significantly differed between patients who presented $(n=27)$ or not $(n=65)$ endocrine toxicity (median: 887 vs 285 days, PFS, $p>0.05 ; 1465$ vs 612 days, OS, $p>0.05$, respectively) (Fig. 2). However, when all toxicities (i.e., including nonendocrine toxicities, Table 5) were considered, PFS and OS were significantly longer in patients who presented irAEs $(n=71)$, compared to those who did not $(n=21)$ (median: 1093 vs 154 days, PFS, $p=0.0002 ; 1200$ vs 320 days, OS, $p=0.0006$, respectively) (Fig. 3), even though in 18 cases immunotherapy had to be temporarily withheld $(n=9)$ or definitively interrupted $(n=9)$.

\section{Discussion}

In this multicentre study, we have addressed irAEs in a large series of cancer patients treated with anti-PD1 agents. We analyzed medical records from 251 patients (155 males and 96 females) affected by different tumors, mainly NSCLC (68.92\%) and melanoma (24.3\%). The median age was 70.6 years, with a range from 44 to 95 years. Patients 
Table 3 Predictive factors for the onset of endocrine toxicity during treatment with nivolumab or pembrolizumab
Table 4 Simultaneous analysis of all possible predictors of endocrine toxicity by binary logistic regression analysis

\begin{tabular}{|c|c|c|c|c|c|c|}
\hline \multirow[t]{2}{*}{ Predictor } & \multicolumn{4}{|c|}{ Toxicity } & \multirow[t]{2}{*}{ Chi2 } & \multirow[t]{2}{*}{$p$} \\
\hline & No & & Yes & & & \\
\hline \multicolumn{7}{|l|}{ Gender } \\
\hline Male & 119 & $76.8 \%$ & 36 & $23.2 \%$ & 4.381 & 0.036 \\
\hline Female & 62 & $64.6 \%$ & 34 & $35.4 \%$ & & \\
\hline \multicolumn{7}{|l|}{ Type of tumor } \\
\hline Head-Neck & 2 & $100.0 \%$ & 0 & $0.0 \%$ & 5.647 & 0.342 \\
\hline Breast & 3 & $75.0 \%$ & 1 & $25.0 \%$ & & \\
\hline Melanoma & 48 & $78.7 \%$ & 13 & $21.3 \%$ & & \\
\hline NSCLC & 120 & $69.4 \%$ & 53 & $30.6 \%$ & & \\
\hline Kidney & 8 & $80.0 \%$ & 2 & $20.0 \%$ & & \\
\hline Bladder & 0 & $0.0 \%$ & 1 & $100.0 \%$ & & \\
\hline \multicolumn{7}{|l|}{ Age } \\
\hline Average age & 70.52 & $\mathrm{n} / \mathrm{a}$ & 70.8 & $\mathrm{n} / \mathrm{a}$ & 0.176 & 0.860 \\
\hline \multicolumn{7}{|c|}{ Nivolumab vs Pembrolizumab } \\
\hline Nivolum ab & 112 & $72.7 \%$ & 42 & $27.3 \%$ & 0.075 & 0.784 \\
\hline Pembrolizumab & 69 & $71.1 \%$ & 28 & $28.9 \%$ & & \\
\hline \multicolumn{7}{|c|}{ Previous endocrine disease } \\
\hline No & 156 & $78.4 \%$ & 43 & $21.6 \%$ & 18.840 & 0.00001 \\
\hline Yes & 25 & $48.1 \%$ & 27 & $51.9 \%$ & & \\
\hline
\end{tabular}

\begin{tabular}{|c|c|c|c|c|c|c|c|c|}
\hline & & \multirow[t]{2}{*}{$\begin{array}{l}\text { Statistical } \\
\text { significance }\end{array}$} & \multirow[t]{2}{*}{ Odd ratio } & \multicolumn{2}{|c|}{$\begin{array}{l}\text { Odd Ratio } \\
\text { C.I. }(95 \%)\end{array}$} & \multirow[t]{2}{*}{ Probability } & \multicolumn{2}{|c|}{$\begin{array}{l}\text { Probability } \\
\text { C.I. }(95 \%)\end{array}$} \\
\hline & & & & Lower & Upper & & Lower & Upper \\
\hline \multirow[t]{2}{*}{ Gender } & Male & 0.074 & 1.717 & 0.95 & 3.103 & 0.233 & 0.215 & 0.250 \\
\hline & Female & & & & & 0.365 & 0.334 & 0.395 \\
\hline \multirow[t]{6}{*}{ Tumor } & NSCLC & 0.284 & 0.799 & 0.53 & 1.204 & 0.298 & 0.277 & 0.320 \\
\hline & Melanoma & & & & & 0.247 & 0.215 & 0.279 \\
\hline & Kidney & & & & & 0.276 & 0.189 & 0.363 \\
\hline & Breast & & & & & 0.320 & 0.177 & 0.464 \\
\hline & Head-Neck & & & & & 0.125 & 0.073 & 0.177 \\
\hline & Bladder & & & & & 0.120 & 0.120 & 0.120 \\
\hline \multirow{2}{*}{$\begin{array}{l}\text { Previous } \\
\text { endocrinopathy }\end{array}$} & No & $<0.001$ & 4.156 & 2.141 & 8.065 & 0.216 & 0.209 & 0.223 \\
\hline & Yes & & & & & 0.539 & 0.518 & 0.560 \\
\hline \multirow[t]{2}{*}{ Anti PD-1 } & Pembrolizumab & 0.902 & 1.039 & 0.57 & 1.894 & 0.289 & 0.260 & 0.318 \\
\hline & Nivolumab & & & & & 0.279 & 0.257 & 0.302 \\
\hline \multirow[t]{6}{*}{ Age } & $\leq 50$ & 0.683 & 0.958 & 0.782 & 1.175 & 0.289 & 0.207 & 0.372 \\
\hline & $51-55$ & & & & & 0.250 & 0.214 & 0.285 \\
\hline & $56-60$ & & & & & 0.278 & 0.217 & 0.340 \\
\hline & $61-65$ & & & & & 0.311 & 0.258 & 0.363 \\
\hline & $66-70$ & & & & & 0.270 & 0.227 & 0.312 \\
\hline & $>70$ & & & & & 0.286 & 0.260 & 0.311 \\
\hline
\end{tabular}

A result is considered statistically significant if it allows to reject the null hypothesis. A significance level of $5 \%$ was considered, namely all the results with a $p$ value $<0.05$ have a 5\% probability that the null hypothesis is correct. Conversely, the same results have a $95 \%$ probability that the expected result is correct C.I. Confidence Interval 
received nivolumab $(61.35 \%)$ or pembrolizumab $(38.65 \%)$ and the follow-up ranged from 2 to 60 months.

Endocrine toxicity was observed in $27.89 \%$ of patients $(n=70)$. Endocrine irAEs are among the most frequent toxicities. An analysis of reports and case series published in the literature from January 2016 to April 2018 retrieved, among the 101 publications included, 139 cases of irAEs and 59 of these $(42.4 \%)$ were identified as endocrine toxicities, including thyroid, adrenal, pancreas alterations [16]. Endocrine alterations may turn out to be complex toxicities and some of them (e.g., hypocortisolism, diabetic ketoacidosis) may be life-threatening, if not recognized and appropriately treated. Data from the FDA Adverse Events Reporting System database revealed 6260 records related to endocrine irAEs during ICPLs treatment. Life-threatening situations occurred in $7.57 \%$ of cases and death in $9.6 \%$ [17].

With regard to the type of tumor in patients presenting endocrine toxicity, the majority of cases were registered in

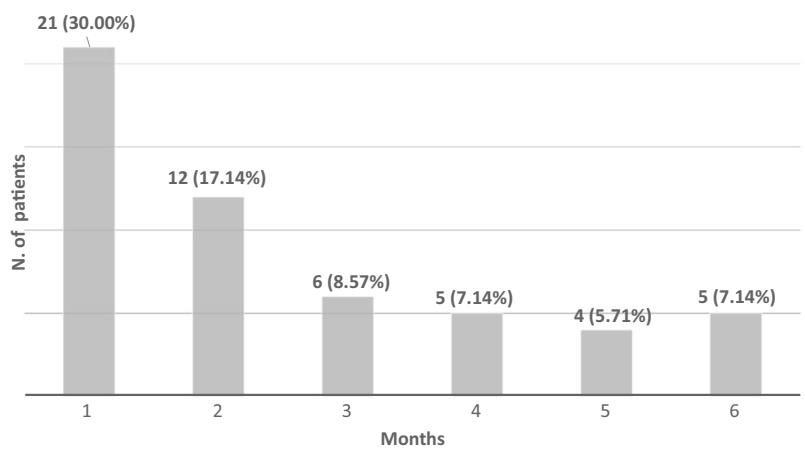

Fig. 1 Time of onset of endocrine toxicity. The \% refers to the total number of patients in which endocrine toxicity was found $(n=70)$

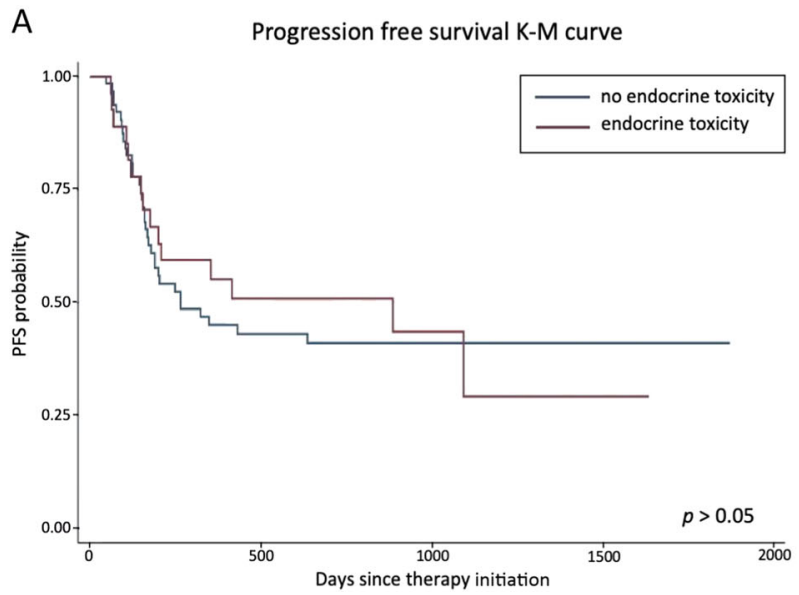

patients with NSCLC (75.71\%) or melanoma (18.57\%), which were the most represented tumors in our database. If we consider the total number of patients with NSCLC or melanoma included in the study, we observed endocrine toxicity in $30 \%$ and $21 \%$ of these tumors, respectively. As per the specific treatment in patients with NSCLC or melanoma, 42 patients $(60 \%)$ were treated with nivolumab and $28(40 \%)$ with pembrolizumab, virtually the same distribution between the two treatments in the total group of 251 patients included in the study.

In almost all cases endocrine irAEs were related to thyroid toxicity $(n=66,94.28 \%)$. In the remaining four cases, we observed two patients who developed diabetes mellitus, which required insulin treatment, and two patients who developed pituitary alterations (secondary hypothyroidism and secondary hypocortisolism). It is likely that hypophysitis caused pituitary dysfunctions in these two patients; however, no MRI or anti-pituitary Abs were available. Anyway, a TC scan of the brain, which included a detailed analysis of the pituitary gland and of the pituitary stalk, excluded the presence of potentially metastatic lesions. These data are in agreement with previously published original studies and reviews, which addressed thyroid toxicity as the most frequent endocrine complication of cancer immunotherapy, particularly with anti-PD1 molecules [10-13]. Interestingly, the thyroid gland appears the preferred target of drugs that modulate the immune response in diseases other than cancer. This is the case for instance of alemtuzumab, a humanized monoclonal $\mathrm{Ab}$ that targets $\mathrm{CD}^{2} 2^{+}$cells, in patients with multiple sclerosis [18]. It has been suggested that the preferential onset of thyropathies during treatment with immunomodulatory drugs may be due to the prevalence of anti-thyroid $\mathrm{Ab}$ in the general population (up to 18\%) and to genetic factors that predispose to thyroid autoimmunity [19].

B

Overall survival K-M curve

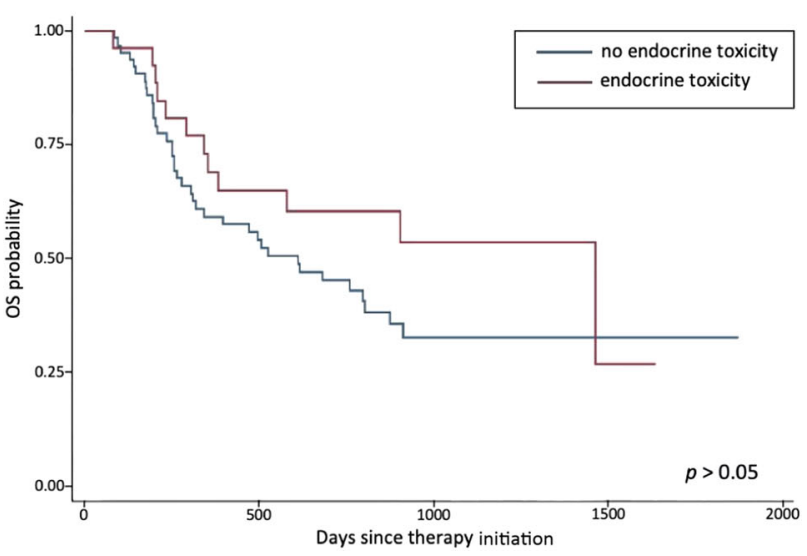

Fig. 2 Association between endocrine toxicity and clinical outcomes. Kaplan-Meier plots of (A) progression-free survival (PFS) and (B) overall survival (OS) in patients who developed endocrine toxicity $v s$ patients who did not 
We then analyzed the presence of possible predictors of endocrine toxicity. We found, in agreement with a previous report, that a pre-existing endocrinopathy is a significant predictor of the development of endocrine irAEs [13]. We also observed a gender difference, with a significantly higher risk to present endocrine toxicity in women. Conversely, the type of cancer, the type of anti PD-1 and age were not predictors of endocrine alterations related to antiPD1 therapy in our series of patients. When these parameters were analyzed simultaneously in a unique statistical model by binary logistic regression, a pre-existing endocrinopathy remained a significant predictor of the occurrence of endocrine toxicity during treatment with nivolumab or pembrolizumab.

An early onset of endocrine toxicity, i.e., within 2 months since the initiation of therapy, was reported in

Table 5 Non-endocrine-related toxicities in the subgroup of patients with NSCLC $(n=92)$ treated with immunotherapy

\begin{tabular}{ll}
\hline Toxicity & Number of cases \\
\hline Fatigue & 31 \\
Skin rash & 18 \\
Itchiness & 16 \\
Diarrhea & 15 \\
Hypertransaminasemia/hyperbilirubinemia & 11 \\
Pneumonia & 10 \\
Hyporexia/anorexia & 10 \\
Arthritis & 8 \\
Mucositis & 7 \\
Fever & 6 \\
Nausea & 6 \\
Myalgia & 4 \\
Xerostomia & 3 \\
\hline
\end{tabular}

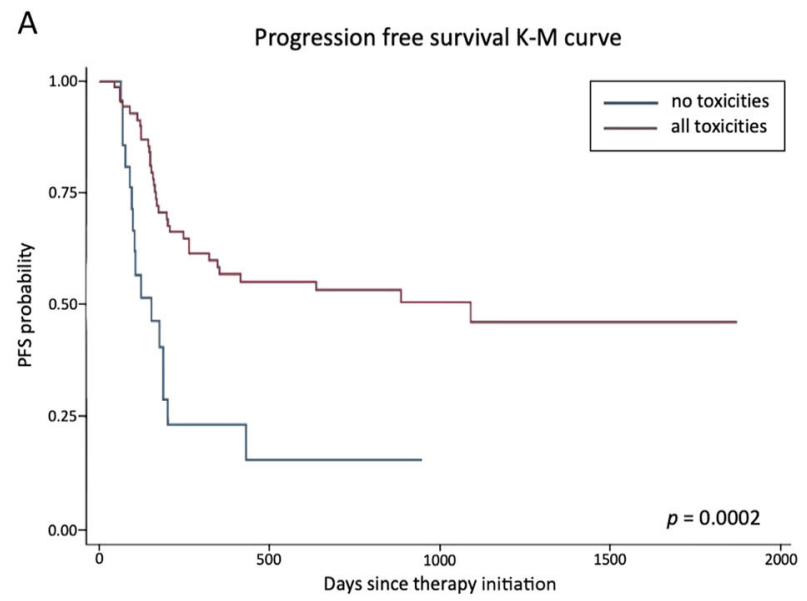

almost half of cases; in three out of four patients endocrine alterations were observed within 6 months. An early occurrence from the first dose of anti-PD1 and the onset of thyroid alterations has been reported in previous studies $[20,21]$. Therefore, it is worth recommending that endocrine alterations, and mostly thyroid dysfunction, should be routinely investigated since the beginning of immunotherapy with anti-PD1 molecules.

As previously mentioned, thyroid alterations were by far the most frequent endocrine irAEs observed in our study. This finding is in agreement with clinical experience, which shows that thyroid disorders are the most common endocrine alterations during cancer treatment with anti PD-1 agents [10]. The most frequent thyroid alteration was represented by hypothyroidism $(51.52 \%)$, followed by thyrotoxicosis or transient thyrotoxicosis with a subsequent shift into hypothyroidism. These three different patterns of thyroid disease presentation have been described in other studies [13, 15, 20-25]. In more than half of patients with a thyropathy $(54.5 \%)$, a mild clinical presentation, which did not require specific treatment, was observed. Conversely, in $45.5 \%$ of cases thyroid specific therapy was required, either as a new therapy or as a dose adjustment of an ongoing treatment.

The etiophathogenesis of thyroid alterations associated with immune-check point inhibitors is still unclear. In particular, it has not been clarified yet whether anti-thyroid Ab play a role in the development of thyroid alterations. This is at least partially due to the fact that anti-thyroid Abs have been rarely determined in the published studies, so far. Admittedly, thyrotoxicosis in this setting is usually mild and transient and there is no recommendation to measure Abs [10]. In our series of patients, anti-thyroid Abs had been checked only in a very few cases and no specific comment is possible. Nevertheless, the presence of thyroid Ab does

B

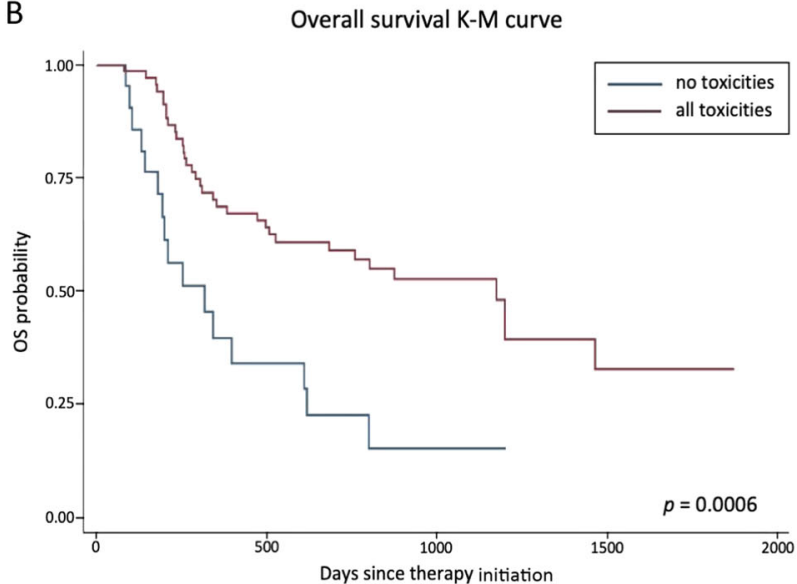

Fig. 3 Association between all toxicities and clinical outcomes. Kaplan-Meier plots of (A) progression-free survival (PFS) and (B) overall survival (OS) in patients who developed toxicities $v s$ patients who did not 
not appear a crucial factor for the development of thyroid dysfunction [25-28], thus suggesting that thyroid toxicity during cancer immunotherapy may be caused by other mechanisms or by other Abs that are not routinely measured [10]. In any case, thyroid autoimmunity in cancer patients treated with ICPIs is worth further investigation, in view of published findings suggesting a more favorable outcome in patients who develop autoimmune features during therapy with immunomodulatory agents (i.e., interferon or GVAX) [29, 30]. With regard to ICPIs, a few studies suggested a better outcome in those patients, in which a thyroid dysfunction occurred during treatment with anti PD-1 or anti PD-L1 molecules, in contrast to our findings [31-33]. However, a clear relationship with thyroid autoimmunity was not demonstrated. In addition, the heterogeneity of the series of patients in different studies (e.g., different types of cancer, different drugs) may likely affect the results of analyses addressing clinical outcomes. Admittedly, this is an interesting, yet not definitively solved, issue, so far.

Noteworthy, when all toxicities, which included symptoms suggesting a possible autoimmune response (e.g., fatigue, skin rash, itchiness, diarrhea, pneumonia, hypertransaminasemia/hyperbilirubinemia, hyporexia/anorexia) were considered, we observed a strong correlation between irAEs and a better outcome, in terms of both PFS and OS.

The fact that our study is retrospective did not allow a formal sample size calculation. Nevertheless, patients had been subjected to a strict follow-up program and the assessment of blood parameters during the scheduled hospital admissions for treatments allowed a standardized collection of data. Admittedly, the main strength of the study relies on the sample size, which represents to our knowledge the largest series of patients included in an original study, so far, outside of a clinical trial protocol, thus representing real-life data.

In summary, in this study we have investigated the presence of endocrine toxicities in a large series of cancer patients treated with the anti-PD1 nivolumab or pembrolizumab. The presence of a pre-existing endocrinopathy was a strong predictor of endocrine toxicity, which in most instances appeared in the first few months after the initiation of immunotherapy. Thyroid dysfunction was by far the most frequent endocrinopathy and both hypothyroidism, preceded or not by transient thyrotoxicosis, or thyrotoxicosis were observed. These alterations required specific pharmacological treatment in almost half of cases. Therefore, endocrine surveillance, and in particular thyroid function assessment, should be routinely performed in these patients.

Acknowledgements The authors wish to thank Dr. Claudio Quinto for technical assistance in statistical analyses.
Funding The authors did not receive support from any organization for the submitted work. Open access funding provided by Université degli Studi di Firenze within the CRUI-CARE Agreement.

\section{Compliance with ethical standards}

Conflict of interest The authors declare no competing interests.

Ethical approval The study was approved by the AOU Careggi ethics committee and was performed in accordance with the ethical standards as laid down in the 1964 Declaration of Helsinki and its later amendments or comparable ethical standards.

Publisher's note Springer Nature remains neutral with regard to jurisdictional claims in published maps and institutional affiliations.

Open Access This article is licensed under a Creative Commons Attribution 4.0 International License, which permits use, sharing, adaptation, distribution and reproduction in any medium or format, as long as you give appropriate credit to the original author(s) and the source, provide a link to the Creative Commons license, and indicate if changes were made. The images or other third party material in this article are included in the article's Creative Commons license, unless indicated otherwise in a credit line to the material. If material is not included in the article's Creative Commons license and your intended use is not permitted by statutory regulation or exceeds the permitted use, you will need to obtain permission directly from the copyright holder. To view a copy of this license, visit http://creativecommons. org/licenses/by/4.0/.

\section{References}

1. F.S. Hodi, S.J. O'Day, D.F. McDermott, R.W. Weber, J.A. Sosman, J.B. Haanen, R. Gonzalez, C. Robert, D. Schadendorf, J. C. Hassel et al. Improved survival with ipilimumab in patients with metastatic melanoma. N. Engl. J. Med. 363, 711-723 (2010)

2. J.D. Wolchok, B. Neyns, G. Linette, S. Negrier, J. Lutzky, L. Thomas, W. Waterfield, D. Schadendorf, M. Smylie, T. Guthrie Jr et al. Ipilimumab monotherapy in patients with pretreated advanced melanoma: a randomised, double-blind, multicentre, phase 2, dose-ranging study. Lancet Oncol. 11, 155-164 (2010)

3. S.L. Topalian, F.S. Hodi, J.R. Brahmer, S.N. Gettinger, D.C. Smith, D.F. McDermott, J.D. Powderly, R.D. Carvajal, J.A. Sosman, M.B. Atkins et al. Safety, activity, and immune correlates of anti-PD-1 antibody in cancer. N. Engl. J. Med. 366, 2443-2454 (2012)

4. A. Ribas, J.D. Wolchok, Combining cancer immunotherapy and targeted therapy. Curr. Opin. Immunol. 25, 291-296 (2013)

5. J. Larkin, V. Chiarion-Sileni, R. Gonzalez, J.J. Grob, C.L. Cowey, C.D. Lao, D. Schadendorf, R. Dummer, M. Smylie, P. Rutkowski et al. Combined Nivolumab and Ipilimumab or Monotherapy in Untreated Melanoma. N. Engl. J. Med. 373, 23-34 (2015)

6. M.A. Postow, M.K. Callahan, J.D. Wolchok, Immune Checkpoint Blockade in Cancer Therapy. J. Clin. Oncol. 33, 1974-1982 (2015)

7. L. Fehrenbacher, A. Spira, M. Ballinger, M. Kowanetz, J. Vansteenkiste, J. Mazieres, K. Park, D. Smith, A. Artal-Cortes, C. Lewanski et al. Atezolizumab versus docetaxel for patients with previously treated non-small-cell lung cancer (POPLAR): a multicentre, open-label, phase 2 randomised controlled trial. Lancet 387, 1837-1846 (2016) 
8. C. Massard, M.S. Gordon, S. Sharma, S. Rafii, Z.A. Wainberg, J. Luke, T.J. Curiel, G. Colon-Otero, O. Hamid, R.E. Sanborn et al. Safety and Efficacy of Durvalumab (MEDI4736), an antiProgrammed cell death ligand-1 immune checkpoint inhibitor, in patients with advanced urothelial bladder cancer. J. Clin. Oncol. 34, 3119-3125 (2016)

9. A. Rittmeyer, F. Barlesi, D. Waterkamp, K. Park, F. Ciardiello, J. von Pawel, S.M. Gadgeel, T. Hida, D.M. Kowalski, M.C. Dols et al. Atezolizumab versus docetaxel in patients with previously treated non-small-cell lung cancer (OAK): a phase 3, open-label, multicentre randomised controlled trial. Lancet 389, 255-265 (2017)

10. L.S. Chang, R. Barroso-Sousa, S.M. Tolaney, F.S. Hodi, U.B. Kaiser, L. Min, Endocrine toxicity of cancer immunotherapy targeting immune checkpoints. Endocr. Rev. 40, 17-65 (2019)

11. R.M. Ruggeri, A. Campennì, G. Giuffrida, P. Trimboli, L. Giovanella, F. Trimarchi, S. Cannavò, Endocrine and metabolic adverse effects of immune checkpoint inhibitors: an overview (what endocrinologists should know). J. Endocrinol. Investig. 42, 199-205 (2019)

12. M. Freeman-Keller, Y. Kim, H. Cronin, A. Richards, G. Gibney, J.S. Weber, Nivolumab in Resected and Unresectable Metastatic Melanoma: characteristics of Immune-Related Adverse Events and Association with Outcomes. Clin. Cancer Res. 22, 886-894 (2016)

13. E.M. Presotto, G. Rastrelli, I. Desideri, V. Scotti, S. Gunnella, N. Pimpinelli, E. Vaccher, A. Bearz, F. Di Costanzo, M. Brugia et al. Endocrine toxicity in cancer patients treated with nivolumab or pembrolizumab: results of a large multicentre study. J. Endocrinol. Investig. 43, 337-345 (2020)

14. P.E. Hughes, S. Caenepeel, L.C. Wu, Targeted Therapy and Checkpoint Immunotherapy Combinations for the Treatment of Cancer. Trends Immunol. 37, 462-476 (2016)

15. A. Jannin, N. Penel, M. Ladsous, M.C. Vantyghem, C. Do Cao, Tyrosine kinase inhibitors and immune checkpoint inhibitors-induced thyroid disorders. Crit. Rev. Oncol. Hematol. 141, 23-35 (2019)

16. R. Bajwa, A. Cheema, T. Khan, A. Amirpour, A. Paul, S. Chaughtai, S. Patel, T. Patel, J. Bramson, V. Gupta et al. Adverse Effects of Immune Checkpoint Inhibitors (Programmed Death-1 Inhibitors and Cytotoxic T-Lymphocyte-Associated Protein-4 Inhibitors): results of a Retrospective Study. J. Clin. Med. Res. 11, 225-236 (2019)

17. Y. Zhai, X. Ye, F. Hu, J. Xu, X. Guo, Y. Zhuang, J. He, Endocrine toxicity of immune checkpoint inhibitors: a real-world study leveraging US Food and Drug Administration adverse events reporting system. J. Immunother. Cancer 7, 286 (2019)

18. L. Scappaticcio, M. Castellana, C. Virili, G. Bellastella, M. Centanni, S. Cannavò, A. Campennì, R.M. Ruggeri, L. Giovanella, P. Trimboli, Alemtuzumab-induced thyroid events in multiple sclerosis: a systematic review and meta-analysis. J. Endocrinol. Investig. 43, 219-229 (2020)

19. R. Percik, Y. Shoenfeld, Check point inhibitors and autoimmunity: why endocrinopathies and who is prone to? Best. Pract. Res. Clin. Endocrinol. Metab. 34, 101411 (2020)

20. D.L. Morganstein, Z. Lai, L. Spain, S. Diem, D. Levine, C. Mace, M. Gore, J. Larkin, Thyroid abnormalities following the use of cytotoxic T-lymphocyte antigen-4 and programmed death receptor protein-1 inhibitors in the treatment of melanoma. Clin. Endocrinol. (Oxf.) 86, 614-620 (2017)
21. E.S. Scott, G.V. Long, A. Guminski, R.J. Clifton-Bligh, A.M. Menzies, V.H. Tsang, The spectrum, incidence, kinetics and management of endocrinopathies with immune checkpoint inhibitors for metastatic melanoma. Eur. J. Endocrinol. 178, 175-182 (2018)

22. H. Lee, F.S. Hodi, A. Giobbie-Hurder, P.A. Ott, E.I. Buchbinder, R. Haq, S. Tolaney, R. Barroso-Sousa, K. Zhang, H. Donahue et al. Characterization of thyroid disorders in patients receiving immune checkpoint inhibition therapy. Cancer Immunol. Res. 5, 1133-1140 (2017)

23. G.C. Leonardi, J.F. Gainor, M. Altan, S. Kravets, S.E. Dahlberg, L. Gedmintas, R. Azimi, H. Rizvi, J.W. Riess, M.D. Hellmann et al. Safety of Programmed Death-1 Pathway Inhibitors Among Patients With Non-Small-Cell Lung Cancer and Preexisting Autoimmune Disorders. J. Clin. Oncol. 36, 1905-1912 (2018)

24. F.X. Danlos, A.L. Voisin, V. Dyevre, J.M. Michot, E. Routier, L. Taillade, S. Champiat, S. Aspeslagh, J. Haroche, L. Albiges et al. Safety and efficacy of anti-programmed death 1 antibodies in patients with cancer and pre-existing autoimmune or inflammatory disease. Eur. J. Cancer 91, 21-29 (2018)

25. J. De Filette, Y. Jansen, M. Schreuer, H. Everaert, B. Velkeniers, B. Neyns, B. Bravenboer, Incidence of thyroid-related adverse events in melanoma patients treated with pembrolizumab. J. Clin. Endocrinol. Metab. 101, 4431-4439 (2016)

26. F. Guaraldi, R. La Selva, M.T. Samà, V. D’Angelo, D. Gori, P. Fava, M.T. Fierro, P. Savoia, E. Arvat, Characterization and implications of thyroid dysfunction induced by immune checkpoint inhibitors in real-life clinical practice: a long-term prospective study from a referral institution. J. Endocrinol. Investig. 41, 549-556 (2018)

27. D.A. Delivanis, M.P. Gustafson, S. Bornschlegl, M.M. Merten, L. Kottschade, S. Withers, A.B. Dietz, M. Ryder, Pembrolizumabinduced thyroiditis: comprehensive clinical review and insights into underlying involved mechanisms. J. Clin. Endocrinol. Metab. 102, 2770-2780 (2017)

28. T. Maekura, M. Naito, M. Tahara, N. Ikegami, Y. Kimura, S. Sonobe, T. Kobayashi, T. Tsuji, S. Minomo, A. Tamiya et al. Predictive factors of nivolumab induced hypothyroidism in patients with non-small cell lung cancer. Vivo 31, 1035-1039 (2017)

29. H. Gogas, J. Ioannovich, U. Dafni, C. Stavropoulou-Giokas, K. Frangia, D. Tsoutsos, P. Panagiotou, A. Polyzos, O. Papadopoulos, A. Stratigos et al. Prognostic significance of autoimmunity during treatment of melanoma with interferon. N. Engl. J. Med. 354, 709-718 (2006)

30. A. De Remigis, T.D. De Gruijl, J.N. Uram, S.C. Tzou, S. Iwama, M.V. Talor, T.D. Armstrong, S.J. Santegoets, S.F. Slovin, L. Zheng et al. Development of thyroglobulin antibodies after GVAX immunotherapy is associated with prolonged survival. Int. J. Cancer 136, 127-137 (2015)

31. J.C. Osorio, A. Ni, J.E. Chaft, R. Pollina, M.K. Kasler, D. Stephens, C. Rodriguez, L. Cambridge, H. Rizvi, J.D. Wolchok et al. Antibody-mediated thyroid dysfunction during T-cell checkpoint blockade in patients with non-small-cell lung cancer. Ann. Oncol. 28, 583-589 (2017)

32. A. Kotwal, L. Kottschade, M. Ryder, Thyroid 30, 177-184 (2020)

33. Y. Zhou, R. Xia, H. Xiao, D. Pu, Y. Long, Z. Ding, J. Liu, X. Ma, Thyroid function abnormality induced by PD-1 inhibitors have a positive impact on survival in patients with non-small cell lung cancer. Int Immunopharmacol. 91, 107296 (2021) 